\title{
The Psychopathology of Basal Ganglia Calcification
}

\author{
MICHAEL P. PHILPOT ${ }^{1}$ and SHON W. LEWIS ${ }^{2}$ \\ 'Department of Psychogeriatrics, United Medical and Dental Schools, Guy's Hospital, London Bridge, \\ London, SE1 9RT, UK \\ ${ }^{2}$ Department of Psychiatry, Charing Cross and Westminster Medical Schools, London, W6 8RF, UK \\ Correspondence: $\operatorname{Dr}$ M. P. Philpot, St. John's House, Hither Green Hospital, Hither Green Lane, \\ London SE13 6RU, UK
}

\begin{abstract}
Basal ganglia calcification (BGC) was found in 36 of 4122 patients undergoing computed tomography as part of a clinical investigation of their psychiatric illness. The prevalence of BGC increased with age in both men and women. No psychiatric diagnosis was specifically associated with BGC although calcification of the putamen and the caudate was only found in patients with functional disorders. No abnormalities of calcium or phosphate metabolism were found. The results do not support the hypothesis that BGC is an aetiological factor in schizophrenia-like psychoses.
\end{abstract}

\section{Introduction}

Basal ganglia calcification (BGC) has been described in association with a number of psychiatric illnesses. Numerous case reports exist (reviewed by Cummings et al., 1983) in which idiopathic BGC has been found in patients with schizophrenia-like psychoses. These patients often exhibit movement disorders of the parkinsonian or choreoathetotic type and many progress to dementia, especially in late-onset cases (Cummings et al., 1983). Francis (1979) described a pedigree in which basal ganglia calcification and psychosis were inherited in an X-linked fashion. Casanova et al. (1989) reported a further family in which schizophrenia was associated with BGC in three members.

Several studies of neurological patients (Brannan et al., 1980; Cohen et al., 1980; Harrington et al., 1981; Koller et al., 1979; Murphy, 1979; Ogata et al., 1987; Sachs et al., 1979; Tuvendran et al., 1982; Kendall and Cavanagh, 1986) have been carried out to determine the clinical significance of BGC as revealed by brain CT. In general, these conclude that BGC is only rarely associated with extrapyramidal signs or calcium dysmetabolism. The overall prevalence of BGC in these studies ranges from 2.4 to 7.5 per 1000 cases but increases with age, the great majority of cases being aged over 50 years.

Cummings et al. (1983) have suggested that widespread BGC, as exemplified by the case they report, might disrupt ascending monoaminergic pathways which pass through the basal ganglia and as a result bring about the neurotransmitter abnormalities associated with schizophrenia. However, 
it is not clear from a review of the case history literature what clinical or aetiological significance one should attach to BGC when found in an individual with psychiatric illness. The present study aims to determine the prevalence of BGC in a psychiatric sample and its possible psychopathological relevance.

\section{Method}

Reports of all CT scans performed on Maudsley Hospital patients between 1977 and 1986 were retrospectively reviewed. During this period an EMI 1010 scanner was in use and a total of 28145 scans were performed. However, this study was concerned with scans performed on psychiatric patients (including those presenting to adult, child, psychogeriatric, substance abuse and forensic services) whose clinical details could be scrutinised. We therefore excluded all scans on patients referred from neurological or neurosurgical services, all patients sent from other hospitals for scan only and any individuals scanned for research purposes. This left 4122 "first-time" and 409 repeat scans. The age and sex of all psychiatric patients receiving scans was recorded.

All tomograms had been assessed by a Consultant Neuroradiologist and those films reported as showing basal ganglia calcification of any degree were retrieved. Direct visual inspection was made by one of us (SWL), blind to any clinical details, to establish the site, laterality and degree of the calcification. Calcification was recorded as involving the right or left globus pallidus, putamen or caudate nucleus or any combination of these. The degree of calcification was rated on a 5-point scale: 0 , absent to 4 , dense (examples are shown in Figs. $1 a, b, \& c$,).

Clinical details were obtained by an independent rater (MP) from case records and analyzed with regard to age, sex, past medical and psychiatric history including family history and previous therapy, duration of illness and details of the present mental state and physical examination with particular emphasis on cogitive and neurological abnormalities. The results of serum calcium, alkaline phosphatase, thyroid function, syphilis serology, ECG and EEG performed at approximately the same time as the CT scan were also recorded.

A control group of patients scanned during the same period without basal ganglia calcification was obtained by determining the next psychiatric patient in the alphabetically arranged index of scan reports who had the same age and sex as each BGC patient. Scans and case records were then analyzed in the same manner as those of the BGC group.

Statistical comparisons between the BGC and control groups were made either by simple chi-square evaluation or analysis of variance. 


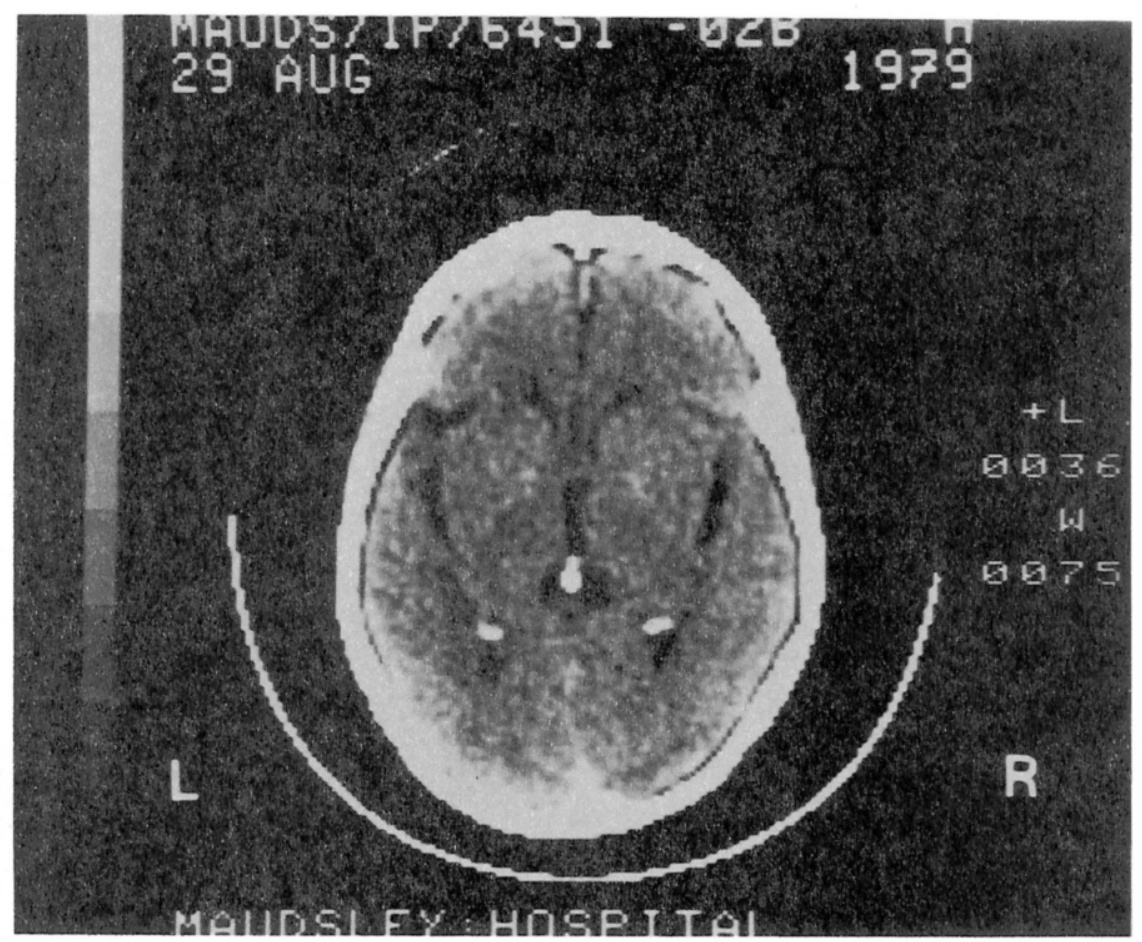

Fig. $1 a$

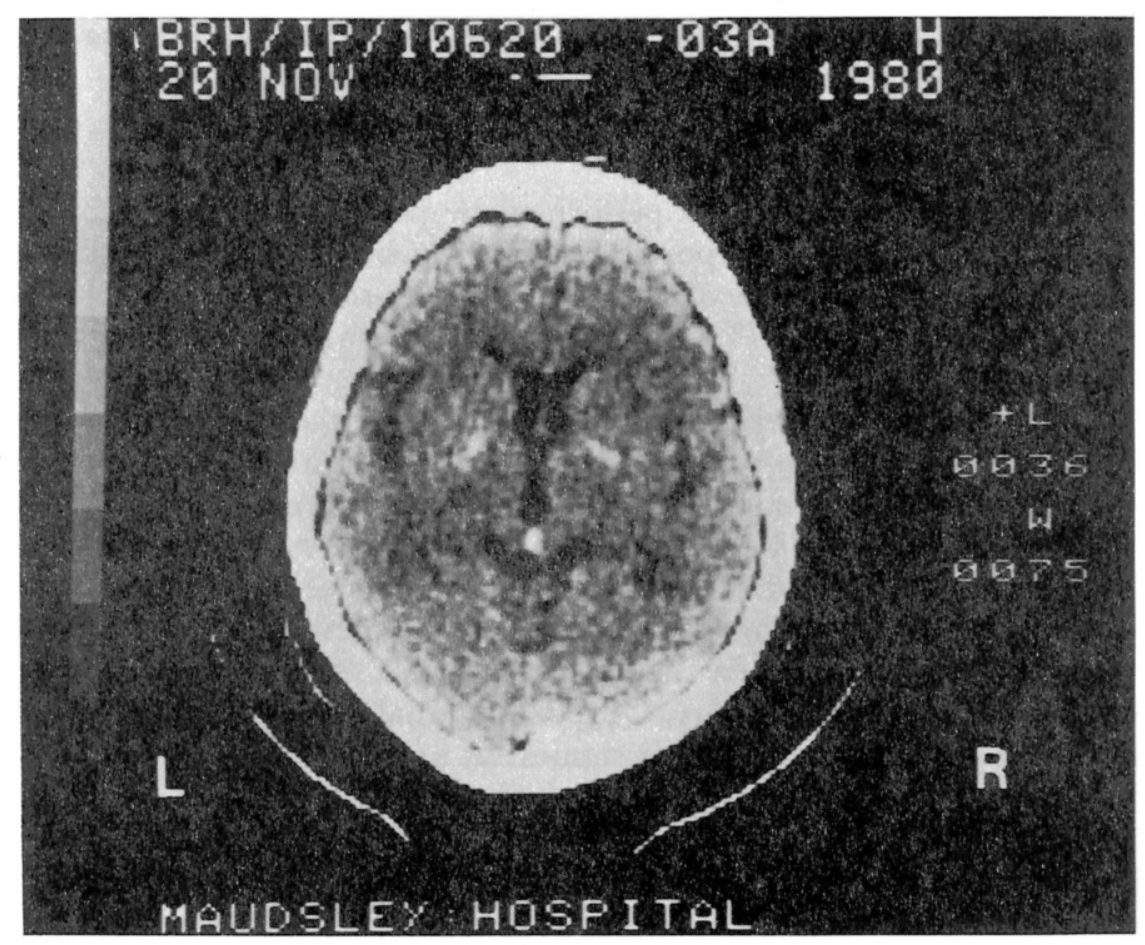

FIg. $1 b$ 


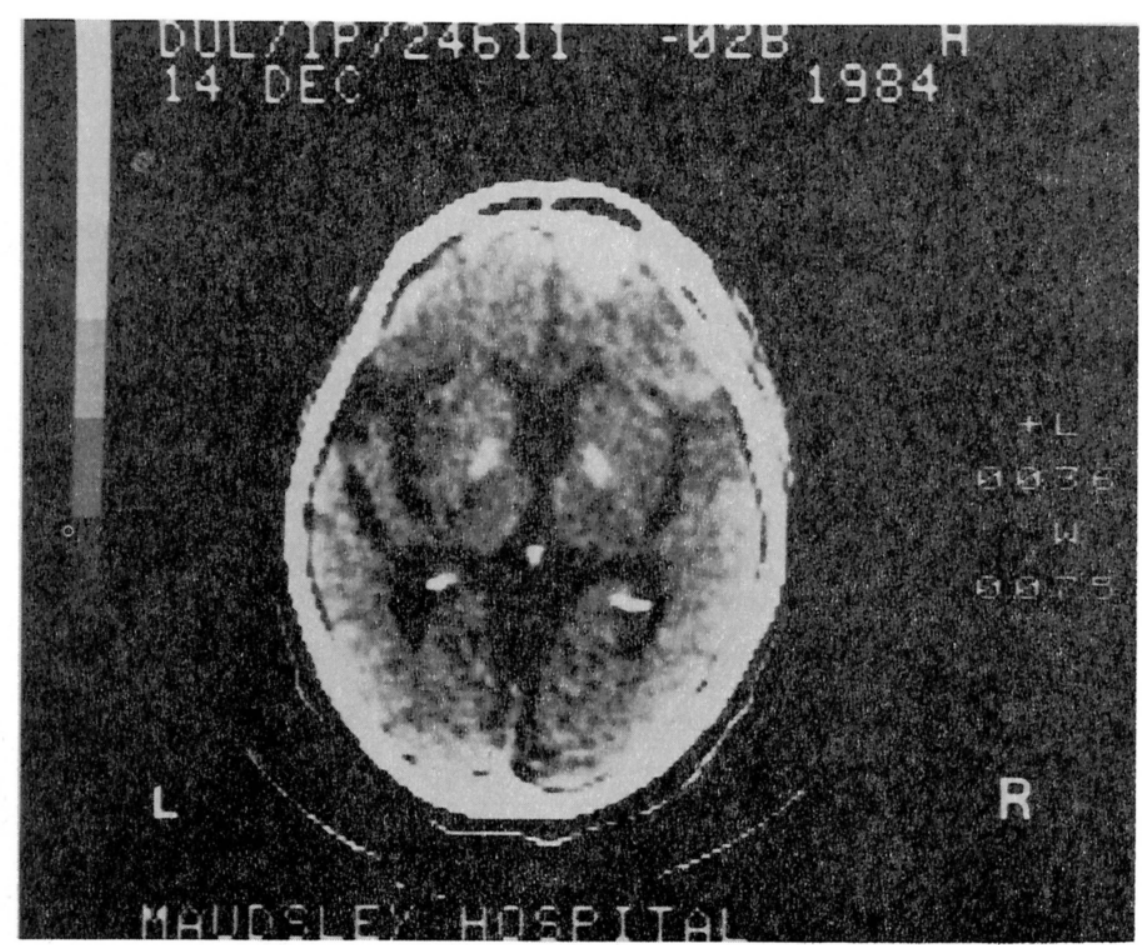

Fig. 1c.

FIG. 1. Examples of globus pallidus calcification of varying severity: (a) faint BGC in a 73year-old woman with depressive anxiety; (b) definite BGC in a 71-year-old woman with vascular dementia; (c) dense BGC in a 60-year-old man with vascular dementia.

\section{Results}

Of the 4122 "first-time" scans performed by psychiatric patients between 1977 and 1986, 36 were reported as showing basal ganglia calcification giving an overall prevalence of 8.7 per 1000 . Sixty-seven $\%$ of the scans were of female patients and $67 \%$ were of patients with functional psychiatric disorders.

Table 1 shows the prevalence of BGC at different ages (i.e. corrected for the total number of scans performed in each age group). BGC appears to be equally prevalent in men and women and increases significantly with age to reach a peak in the 65-74 age group (chi sq $=62.8, \mathrm{df}=3, p<0.0001)$. Table 2 lists the diagnoses found in the BGC group in comparison to those found in the controls. No particular diagnoses were associated with BGC and there were no differences between the groups in terms of family history or past medical and psychiatric history. Of the individual clinical features found on mental state examination the only significant findings were that the percentage of patients showing memory impairment and dysphasia was greater in the control group ( 39 vs $67 \%$ and 14 vs $36 \%$ ). The frequency of abnormal laboratory test results did not differ between BGC and controls, neither did the duration of the psychiatric illness or previous treatment history. 
THE PSYGHOPATHOLOGY OF BASAL GANGLIA CALCIFICATION

TABLE 1. Prevalence of basal ganglia calcification in different age groups

\begin{tabular}{lrrrrc}
\hline & \multicolumn{5}{c}{ Age group } \\
\cline { 2 - 6 } & $0-44$ & $45-64$ & $65-74$ & $75-84$ & $85+$ \\
\hline Male cases per 1000 scans & 1.56 & 5.67 & 30.49 & 9.71 & - \\
Female cases per 1000 scans & 1.12 & 7.56 & 34.38 & 28.23 & 13.89 \\
\hline
\end{tabular}

TABLE 2. Sex, age and diagnoses of subjects with basal ganglia calcification (BGC) and controls

\begin{tabular}{|c|c|c|c|}
\hline & $\begin{array}{c}B G C \\
n=36\end{array}$ & \multicolumn{2}{|c|}{$\begin{array}{c}\text { Control } \\
n=36\end{array}$} \\
\hline$\%$ female & 67 & \multicolumn{2}{|c|}{67} \\
\hline Mean age-males & $60 \pm 15$ & \multicolumn{2}{|c|}{$61 \pm 16$} \\
\hline Mean age-females & $71 \pm 10$ & \multicolumn{2}{|c|}{$72 \pm 9$} \\
\hline \multicolumn{4}{|l|}{ Diagnosis } \\
\hline & $\%$ & $\%$ & $\mathbf{n}$ \\
\hline Primary dementias & 11 & 22 & 8 \\
\hline Vascular dementias & 22 & 31 & 11 \\
\hline Manic depressive psychosis & $33 \quad 12$ & 17 & 6 \\
\hline Schizophrenias & 11 & 14 & 5 \\
\hline Neuroses & 17 & 8 & 3 \\
\hline Personality disorders (inc. alcoholism) & 6 & 8 & 3 \\
\hline
\end{tabular}

TABLE 3. Relationship between site of calcification and diagnosis

\begin{tabular}{lcc}
\hline & \multicolumn{2}{c}{ Site of calcification } \\
\cline { 2 - 3 } & $\begin{array}{c}\text { Globus pallidus only } \\
n=20\end{array}$ & $\begin{array}{c}\text { Putamen/caudate } \\
\pm \text { globus pallidus } \\
n=16\end{array}$ \\
\hline Dementia present & 12 & 0 \\
Dementia absent & 8 & 16 \\
\hline
\end{tabular}

Chi-square with Yates' correlation $=11 \cdot 7(p<0 \cdot 001)$

Twenty scans showed calcification of the globus pallidus alone (including five with unilateral calcification). The remaining 16 had calcification elsewhere; four in the putamen alone, six in the caudate alone, and six in putamen, caudate and globus pallidus. Table 3 shows how the psychiatric diagnosis varied with the site of the calcification, dementia being signifi- 
cantly associated with calcification of the globus pallidus. Calcification of the putamen and caudate was only found in patients with functional psychiatric disorders: eight of whom had affective psychosis, three schizophrenia and five neurotic disorders. There were no correlations between severity of calcification and age, diagnosis, duration of illness or calcium and alkaline phosphatase levels.

\section{Discussion}

Basal ganglia calcification (BGC) has been described in association with a wide variety of conditions including parathyroid disease, hypothyroidism, epilepsy, Parkinson's disease and Down's syndrome (Lowenthal and Bruyn, 1968). It may also occur in patients who have sustained brain damage following anoxia, lead and carbon monoxide poisoning, intracranial infection such as congenital rubella, toxoplasmosis, brucellosis (Mousa et al., 1987) and AIDS (Belman et al., 1986) and the administration of methotrexate or cerebral radiotherapy (Murphy, 1979).

BGC may be identified by skull X-ray or computed tomography (CT) and while the latter method is more sensitive, the former may have greater clinical significance: $70-80 \%$ of BGC visualized by skull X-ray may be associated with hypothyroidism (Bennett et al., 1959; Lowenthal and Bruyn).

Despite the large number of conditions and circumstances which may be associated with CT-visualized BGC the majority of cases are idiopathic. Such cases may be sporadic or familial. Familial forms, so-called Fahr's disease, can show X-linked dominant (Francis, 1979) or autosomal dominant (Moskowitz et al., 1971; Boller et al., 1977) transmission.

In the present study, reviewing 4122 consecutive CT scans of psychiatric patients, we found the prevalence of reported BGC to be $8 \cdot 7$ per 1000 . This is a similar figure to that previously found in neurological populations. The risk of BGC increased with age such that the rate in patients over 65 was approximately five times that in young adults. No significant differences in diagnostic grouping were found between the 36 patients with BGC and 36 age and sex matched controls. Calcification of the putamen and caudate was confined to patients without dementia, most of whom were elderly.

Brannan et al. (1980) have suggested a subdivision of BGC into "senescent" and "secondary acquired" forms, "senescent" BGC being by far the most common and evolving as a result of vascular damage within the basal ganglia occurring in conjunction with the disturbances in calcium and alkaline phosphatase metabolism which themselves increase with advancing age. In addition, it has been postulated that this form chiefly affects the globus pallidus and is bilateral and symmetrical (Koller et al., 1979; Cohen et al., 1980). Unilateral BGC or calcification affecting other parts of the basal ganglia, such as the putamen or caudate nucleus, may indicate a more pathological process.

In conclusion, the results of this study offer little support for the hypothesis that BGC is an important aetiological factor in schizophrenia- 
like psychoses (Cummings et al., 1983). The presence of BGC on CT scans shows little diagnostic specificity, although it is more common, particularly in the globus pallidus, in old age. Pedigrees in which BGC is associated with functional disorders are likely to be extreme rarities.

\section{References}

Belman, A. L., Lantos, G., Horoupian, D. et al. (1986) AIDS: Calcification of the basal ganglia in infants and children. Neurology, 36, 1192-1199.

Boller, F., Boller, M. and Gilbert, J. (1977). Familial idiopathic cerebral calcification. Fournal of Neurology, Neurosurgery and Psychiatry, 40, 280-285.

Bennett, J. C., Maffley, R. H. and Steinbach, H. L. (1959). The significance of bilateral basal ganglia calcification. Radiology, 72, 368-377.

Brannan, T. S., Burger, A. A. and Chaudhury, M. Y. (1980). Bilateral basal ganglia calcification visualised on CT scan. Fournal of Neurology, Neurosurgery and Psychiatry, 43, 403-406.

Casanova, M. F., Hyde, T., Carosella, N. et al. (1989). Concordance of familial nonarteriosclerotic mineralization of the basal ganglia and schizophrenia in two generations: a case report. Schizophrenia Research, 134, (abstract).

Cohen, C. R., Weinstein, M. A. and Duchesneau, P. M. (1980). Calcification of the basal ganglia as visualised by computer tomography. Radiology, 134, 97-99.

Cummings, J. L., Gosenfeld, L. F. Houlihan, J. P. et al. (1983). Neuropsychiatric disturbances associated with idiopathic calcification of the basal ganglia. Biological Psychiatry, 18, 591-601.

Francis, A. F. (1979). Familial basal ganglia calcification and schizophreniform psychosis. British Journal of Psychiatry, 135, 360-362.

Harrington, M. G., McPherson, P., McIntosh, W. B. et al. (1981). The significance of the incidental finding of basal ganglia calcification on computed tomography. Fournal of Neurology, Neurosurgery and Psychiatry, 44, 1168-1170.

Kendall, B. and Cavanagh, N. (1986). Intracranial calcification in paediatric computed tomography. Neuroradiology, 28, 324-330.

Koller, W. C., Cochran, J. W. and Klawans, H. L. (1979). Calcification of the basal ganglia: computerised tomography and clinical correlation. Neurology, 29, 328-333.

Lowenthal, A. and Bruyn, G. W. (1968). Calcification of the striatopallidodentate system. In "Handbook of Clinical Neurology." Vol. 16 (Eds G. W. Bruyn and P. Vinken). North Holland, Amsterdam, pp. 703-723.

Moskowitz, M. A., Winickoff, R. N. and Heinz, E. R. (1971). Familial calcification of the basal ganglia: a metabolic and genetic study. New England Journal of Medicine, 285, 7277.

Mousa, A. M., Muhtaseb, S. A., Reddy, R. R. et al. (1987). The high rate of prevalence of CT-detected basal ganglia calcification in neuropsychiatric (CNS) brucellosis. Acta Neurologica Scandinavica, 76, 448-456.

Murphy, M. J. (1979). Clinical correlations of CT scan detected calcification of the basal ganglia. Annals of Neurology, 6, 507-511.

Ogata, A., Ishida, S. and Wada, T. (1987). A survey of 37 cases with basal ganglia calcification (BGC): CT scan findings of BGC and its relationship to underlying diseases and epilepsy. Acta Neurologica Scandinavica, 75, 117-124.

Sachs, G., Ericson, K., Erasmie, U. et al. (1979). Incidence of basal ganglia calcification on computed tomography. Fournal of Computer Assisted Tomography, 3, 339-344.

Tuvenendran, K., Low, C. H., Boey, H. K. et al. (1982). Basal ganglia calcification on computer tomographic scan: a clinical and radiological correlation. Acta Neurological Scandinavica, 66, 309-315. 


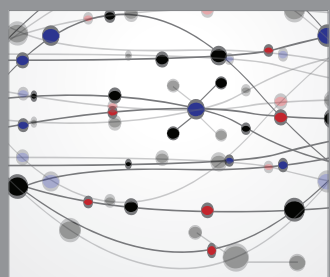

The Scientific World Journal
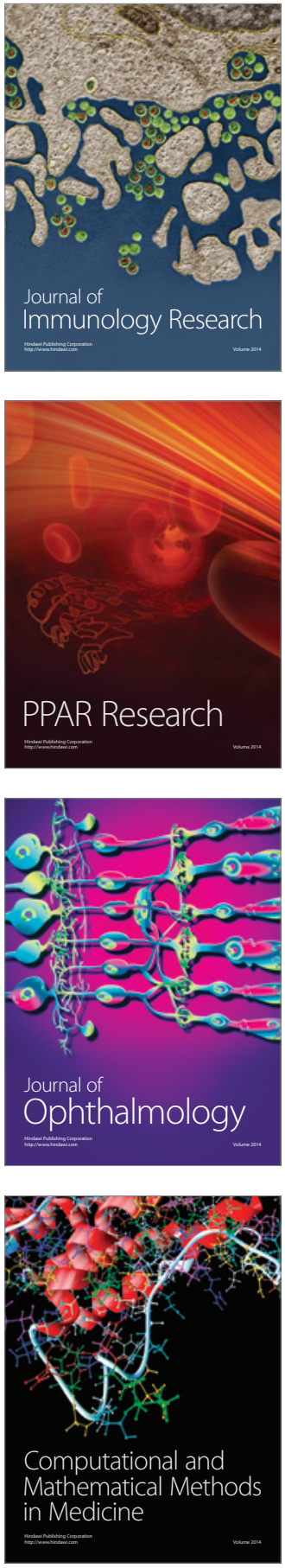

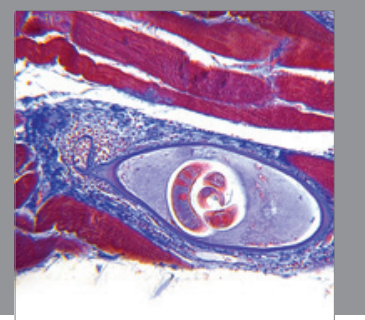

Gastroenterology

Research and Practice
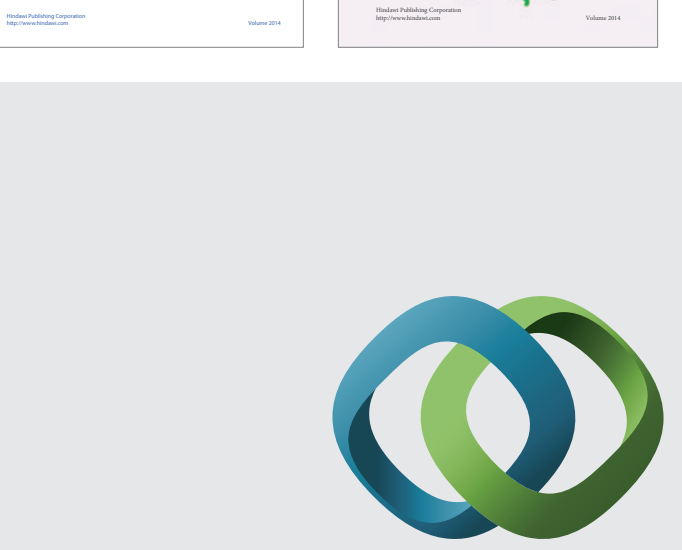

\section{Hindawi}

Submit your manuscripts at

http://www.hindawi.com
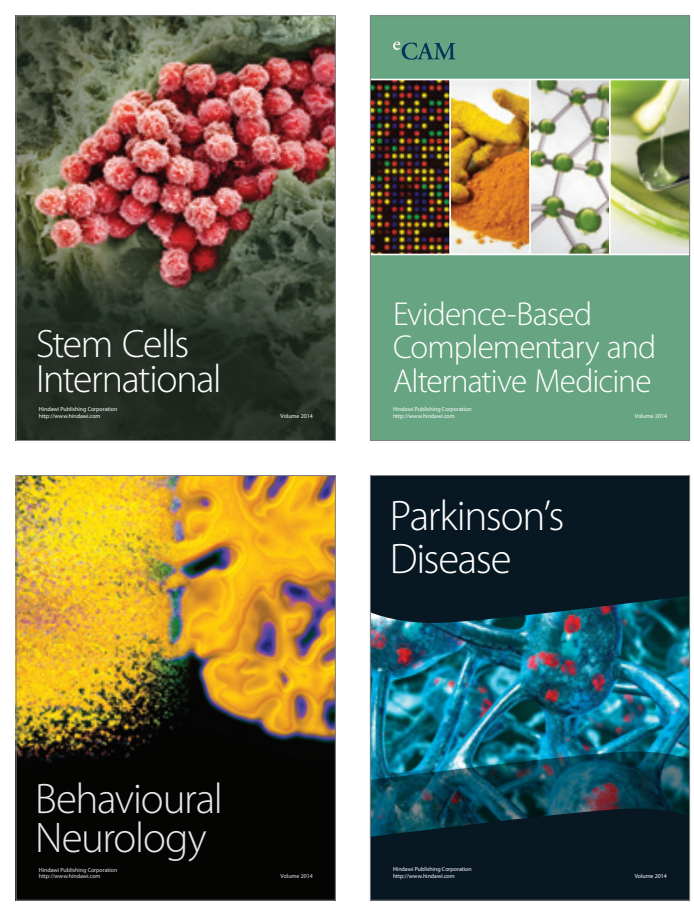

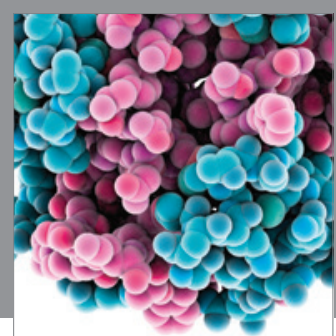

Journal of
Diabetes Research

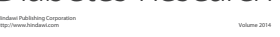

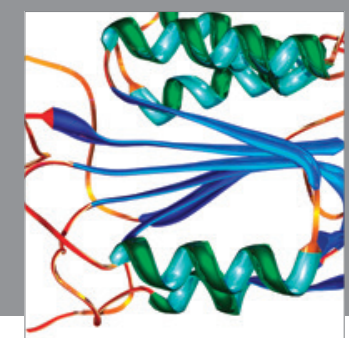

Disease Markers
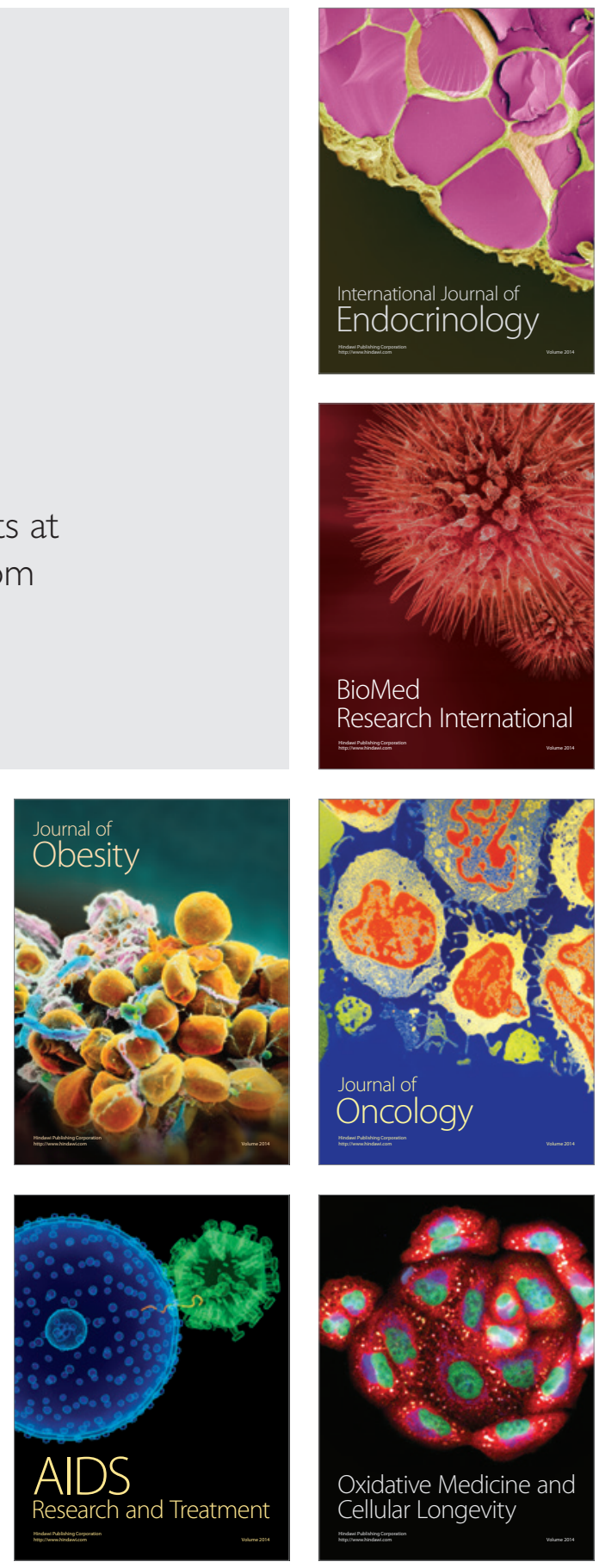